\title{
Unconventional phases of attractive Fermi gases in synthetic Hall ribbons
}

\author{
Sudeep Kumar Ghosh, ${ }^{1, *}$ Sebastian Greschner, ${ }^{2}$ Umesh K. Yadav, ${ }^{3}$ Tapan Mishra, ${ }^{4}$ Matteo Rizzi, ${ }^{5}$ and Vijay B. Shenoy ${ }^{1}$ \\ ${ }^{1}$ Centre for Condensed Matter Theory, Department of Physics, Indian Institute of Science, Bangalore 560012 , India \\ ${ }^{2}$ Institut für Theoretische Physik, Leibniz Universität Hannover, 30167 Hannover, Germany \\ ${ }^{3}$ Department of Physics, Ewing Christian College, Allahabad - 211003, India \\ ${ }^{4}$ Department of Physics, Indian Institute of Technology, Guwahati, Assam 781039, India \\ ${ }^{5}$ Institut für Physik, Universität Mainz, Staudingerweg 7, D-55099 Mainz, Germany
}

(Received 7 November 2016; published 19 June 2017)

\begin{abstract}
An innovative way to produce quantum Hall ribbons in a cold atomic system is to use $M$ hyperfine states of atoms in a one-dimensional optical lattice to mimic an additional "synthetic dimension." A notable aspect here is that the $\mathrm{SU}(M)$ symmetric interaction between atoms manifests as "infinite ranged" along the synthetic dimension. We study the many-body physics of fermions with $\mathrm{SU}(M)$ symmetric attractive interactions in this system using a combination of analytical field theoretic and numerical density-matrix renormalization-group methods. We uncover the rich ground-state phase diagram of the system, including unconventional phases such as squished baryon fluids, shedding light on many-body physics in low dimensions. Remarkably, changing the parameters entails interesting crossovers and transition; e.g., we show that increasing the magnetic field (that produces the Hall effect) converts a "ferrometallic" state at low fields to a "squished baryon superfluid" (with algebraic pairing correlations) at high fields. We also show that this system provides a unique opportunity to study quantum phase separation in a multiflavor ultracold fermionic system.
\end{abstract}

DOI: 10.1103/PhysRevA.95.063612

\section{INTRODUCTION}

Enhanced interest in the field of low dimensional systems such as graphene, nanowires, and two-dimensional (2D) topological insulators has been motivated by their potential application in developing nanoscale devices. Topology plays an important role in such systems and gives rise to several exotic phases such as topological superfluids with Majorana bound states [1-3]. Some of the crucial ingredients in generating topological phases include parameters such as spin-orbit coupling and external magnetic fields. Very fast paced advances in cold atomic systems in employing synthetic gauge fields [4-6] in neutral atomic systems provide realization of such ingredients with unparalleled control over the parameter space, thus pushing beyond the possibilities of the traditional solid-state setups. In particular, cold atoms in optical lattices have been used to engineer topological band structures [7-10] and unconventional phases of matter with nontrivial topology [4,11]. Remarkable flexibility of these systems offers possibilities of manipulation even at the single site level [12,13].

The recent realization of the "synthetic dimension" [14] system in a one-dimensional (1D) optical lattice is one such example of the use of cold atoms as a promising platform, among other things, to realize topological states of matter. A synthetic dimension (SD) system is created by coupling the $M$ hyperfine states of multiflavor atoms loaded in a $1 \mathrm{D}$ optical lattice via Raman transition and thus using the hyperfine space of the atoms as an extra dimension. This system provides a realization of an optical flux lattice in a finite strip with tunable flux per plaquette or equivalently a Hall ribbon. By an appropriate choice of the flux, therefore, the Hofstadter model [15] in a finite square lattice strip is realized in this

\footnotetext{
*sudeep.iisc@gmail.com
}

system. Recent experiments [16-18] with both bosonic and fermionic particles have realized this system with interesting developments such as the observation of chiral edge states.

Alkaline-earth atoms, which are particularly suited for the realization of the SD system, are characterized by a filled-shell electronic configuration. This donates to them two remarkable features: (i) the existence of two metastable states that ease considerably Raman-assisted schemes [17] and (ii) the completely $\mathrm{SU}(M)$ symmetric nature of contact interactions between the $M$ possible orientations of their nuclear spin [19-21]. Experimental developments [22-24] in realizing such large spin particles in cold atoms have made it possible to achieve control over their interactions by, for example, orbital Feshbach resonances [25-27]. Hence, both repulsive and attractive interactions can be generated between particles moving in the SD. One particular important aspect of such interaction is that it is long-ranged along the SD-any two of the hyperfine states can interact with each other with the same strength. These interesting possibilities have triggered an intense theoretical research activity with repulsive interactions in fermionic [28,29] and in bosonic [30,31] SD systems. In this paper, we consider attractive SU $(M)$ interaction (see Refs. [3234] for other studies) between fermions moving in the SD.

A system of multiflavor fermions in a 1D optical lattice with $\mathrm{SU}(M)$ symmetric attractive interaction but without Raman couplings has been well studied [35-40]. The ground state is a fluid of $M$-body bound states of fermions [SU(M) singlets] that are dubbed as "baryons," in analogy with similar $M$-body $\mathrm{SU}(M)$ singlets, e.g., a SU(3) proton, arising in high-energy physics. With Raman couplings, the SD system is a Hall ribbon with infinite ranged interaction along the synthetic dimension. The key open question is, what are the many-body phases of fermions in this system? The goal of this paper is to elucidate this outstanding issue. The attractive interaction makes it clear that the ground state of the system is made of baryons, but their nature is to be discovered. Using 
a combination of field theoretic and numerical density-matrix renormalization-group (DMRG) methods, we uncover the rich phase diagram of the system. Interestingly, there appears a variety of phases including ferrometallic fluids, squished baryon fluids, and more. There are also intriguing crossovers and transitions that are quite unusual: a ferrometal (generalized spin-polarized Fermi fluid) at very small magnetic flux (flux per plaquette close to zero) is converted to a squished baryon quasisuperfluid (algebraically correlated quasicondensate of nonlocal pairs or nonlocal baryons) by increasing the magnetic field ( $\pi$ flux per plaquette). Furthermore, we observe a regime of macroscopic quantum phase separation [41] pointing to the possibilities of the SD system to address a variety of issues in condensed matter.

The rest of the paper is organized as follows. In Sec. II, we describe the Hamiltonian of the SD system with $\mathrm{SU}(M)$ symmetric attractive interaction and discuss that the manybody physics of the system is best analyzed by moving to a different basis. Then, we first consider several limiting cases in which analytical results are possible in Sec. III. Of particular interest is the description of the system for the case of $\pi$ flux with large Raman coupling strength in terms of effective spin- $\frac{1}{2}$ particles and its corresponding field theoretic description. Section IV contains detailed discussion of our numerical DMRG results for the $M=2$ and 3 cases. Finally, in Sec. V, we summarize our results and discuss possibilities of characterizing the unconventional phases of the SD system in cold atom experiments.

\section{DESCRIPTION OF THE SYSTEM}

An SD system created by coherently coupling $M$ hyperfine states of atoms loaded in a 1D optical lattice via Raman lasers can be described by a single-particle Hamiltonian

$$
H_{0}=-t \sum_{i ; \gamma=1}^{M} C_{i+1, \gamma}^{\dagger} C_{i, \gamma}+\sum_{i ; \gamma=1}^{M-1} \Omega_{\gamma}^{i} C_{i, \gamma+1}^{\dagger} C_{i, \gamma}+\text { H.c. }
$$

where $C_{i, \gamma}^{\dagger}$ and $C_{i, \gamma}$ are the fermion operators associated with site $i$ of the optical lattice (coordinate $x_{i}=i d, d$ is the lattice spacing) and hyperfine state $\gamma$. The hopping amplitude from the $i$ th site to its nearest neighbor is $t . \Omega_{\gamma}^{i}=\Omega_{\gamma} e^{-i k_{l} x_{i}}\left(k_{l}\right.$ is the wave vector of the Raman laser) is the Raman coupling of the hyperfine state $\gamma$ with $(\gamma+1)$ and its phase results in an effective magnetic flux $\phi=k_{l} d$ per plaquette of the synthetic lattice. The attractive $\mathrm{SU}(M)$ symmetric interaction of strength $U$ ( $>0$ ) between particles moving in the SD is given by

$$
H_{U}=-\frac{U}{2} \sum_{i, \gamma, \gamma^{\prime}} C_{i, \gamma}^{\dagger} C_{i, \gamma^{\prime}}^{\dagger} C_{i, \gamma^{\prime}} C_{i, \gamma} .
$$

The physics of the SD system is most transparently viewed in another basis ("flavor" basis labeled by $\zeta$ ). The fermion operators in this basis, defined as $\mathbb{b}_{i} \equiv\left\{b_{i, \zeta} ; \zeta=1, \ldots, M\right\}^{T}$, are unitarily $[33,42]$ related to the original operators, $\mathbb{C}_{i} \equiv$ $\left\{C_{i, \gamma} ; \gamma=1, \ldots, M\right\}^{T}$, by $\mathbb{C}_{i}=\mathbb{U}_{i} \mathbb{b}_{i}$, where $\mathbb{U}_{i}$ is a unitary matrix. This $\mathbb{U}_{i}$ matrix can be further decomposed into two matrices: $\mathbb{U}_{i}=\mathbb{W}_{i} \mathbb{S}$. Here, $\mathbb{W}_{i}=\operatorname{Diag}\left\{e^{i k_{l}^{\gamma} x_{i}} ; \gamma=1, \ldots, M\right\}$ with $k_{l}^{\gamma}=(\gamma-1) k_{l}$ is a diagonal matrix containing all the position dependence of $\mathbb{U}_{i}$ and $\mathbb{S}$ is a unitary matrix which

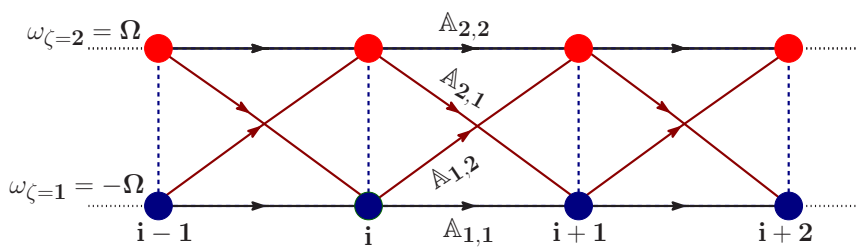

FIG. 1. Kinetic energy: Schematic plot of the $M=2$ SD system with $\mathrm{SU}(2)$ gauge and Zeeman field. $\mathbb{A}_{\zeta, \zeta}$ are the flavor preserving hoppings and $\mathbb{A}_{1,2}$ and $\mathbb{A}_{2,1}$ are the flavor-orbital couplings. Note that the $\zeta$ states at any site $i$ are Zeeman split and there is no hopping between them.

is position independent. In this basis, the total Hamiltonian $\mathcal{H}=H_{0}+H_{U}$ of the system can be recast as

$$
\mathcal{H}=-t \sum_{i}\left(\mathbb{b}_{i+1}^{\dagger} \mathbb{A}_{i}+\text { H.c. }\right)+\sum_{i} \mathbb{b}_{i}^{\dagger} \omega \mathbb{b}_{i}+H_{U},
$$

where $H_{U}$ is the same as that in Eq. (2) with $C_{i \gamma}$ replaced by $b_{i \zeta}$. The SD system is then reduced to a system of $M$ component fermions experiencing a non-Abelian $\mathrm{SU}(M)$ gauge field encoded in the matrix $\mathbb{A}=\mathbb{U}_{i+1}^{\dagger} \mathbb{U}_{i}$ and an $\mathrm{SU}(M)$ "Zeeman field" $\omega=\operatorname{Diag}\left\{\omega_{\zeta} ; \zeta=1, \ldots, M\right\}$ (a diagonal matrix with eigenvalues $\omega_{\zeta}$ ). Interestingly, $\mathbb{A}$ is independent of the position $i$ and can be further simplified as $\mathbb{A}=\mathbb{S}^{\dagger} \mathbb{O S}$ where the position independent diagonal matrix $\mathbb{O}=\operatorname{Diag}\left\{e^{i \phi(\gamma-1)} ; \gamma=\right.$ $1, \ldots, M\}$. The $\mathrm{SU}(M)$ gauge field produces a flavor orbital coupling, i.e., the quantum number $\zeta$ can be altered by hopping from site $i$ to its neighbor and is characterized by the nondiagonal elements of $\mathbb{A}$. The Raman coupling strengths $\Omega_{\gamma}$ depend on the details of a particular experimental realization $[14,16,17]$. To illustrate the results, here we consider $\Omega_{\gamma}=\Omega$ (independent of $\gamma$ ) but a similar analysis can be readily adopted for a general case. For this case, explicit expressions for the $\mathbb{S}$ matrix and the $\omega$ matrix are given by $S_{\gamma, \zeta}=\sqrt{\frac{2}{M+1}} \sin \left(\frac{\pi}{M+1}(M-\gamma+1)(M-\zeta+1)\right)$ and $\omega_{\zeta}=$ $-2 \Omega \cos \left(\frac{\pi \zeta}{M+1}\right)$, respectively. Consequently, as examples, the explicit expression for the $\mathbb{A}$ matrix for the $M=2$ case is

$$
\mathbb{A}=e^{i \frac{\phi}{2}}\left(\begin{array}{cc}
\cos \left(\frac{\phi}{2}\right) & i \sin \left(\frac{\phi}{2}\right) \\
i \sin \left(\frac{\phi}{2}\right) & \cos \left(\frac{\phi}{2}\right)
\end{array}\right)
$$

and for the $M=3$ case is

$$
\mathbb{A}=\left(\begin{array}{ccc}
e^{i \phi} \cos ^{2}\left(\frac{\phi}{2}\right) & \frac{e^{2 i \phi}-1}{2 \sqrt{2}} & -e^{i \phi} \sin ^{2}\left(\frac{\phi}{2}\right) \\
\frac{e^{2 i \phi}-1}{2 \sqrt{2}} & e^{i \phi} \cos (\phi) & \frac{e^{2 i \phi}-1}{2 \sqrt{2}} \\
-e^{i \phi} \sin ^{2}\left(\frac{\phi}{2}\right) & \frac{e^{2 i \phi}-1}{2 \sqrt{2}} & e^{i \phi} \cos ^{2} \frac{\phi}{2}
\end{array}\right) .
$$

A schematic illustration of the kinetic energy of the SD system for $M=2$ is shown in Fig. 1. Note that there are no onsite "spin-flip" terms and for an arbitrary $\phi$ each $\zeta$ flavor is not individually conserved. As a result, the system does not have a global $\mathrm{SU}(M)$ symmetry-only a U(1) symmetry corresponding to the overall particle number conservation survives.

We analyze this model using analytical methods including a field theoretic description [43-45] applied to an effective Hamiltonian (for $\phi=\pi$ ) that we construct and exact numerical 
methods such as DMRG [46-48]. We define the density $n$ of the system as $n=N /(L M)$, which is the average number of particles per site of the synthetic lattice having $L$ optical lattice sites with $N$ particles, and throughout this paper we focus on $n \lesssim 1 / M$.

\section{ANALYTICAL RESULTS}

The parameter space of the SD system for any $M$ is spanned by the three dimensionless parameters: $t / U, \Omega / U$, and $\phi$ (setting $U$ as the basic scale). We note that there is an important energy scale in the system arising due to the competition between the Zeeman energy and the interaction energy. This scale is determined by the critical Raman coupling strength $\Omega_{c}$ at which an $M$-baryon is completely broken by the Zeeman field. In the limit of $t=0, \Omega_{c}=\Omega_{c}^{0}=\frac{M-1}{4 \cos \left(\frac{\pi}{M+1}\right)} U$ [since the energy of an $M$-baryon is $-\frac{M(M-1)}{2} U$ and that of a completely broken $M$-baryon is $\left.-2 M \Omega_{c}^{0} \cos \left(\frac{\pi}{M+1}\right)\right]$ and in general for $t \neq 0, \Omega_{c} \leqslant \Omega_{c}^{0}$.

In the following, we discuss analytical results possible for $t / U \lesssim 1$ in the limit of very small and very large values of $\Omega$. These two limits become analytically accessible because of the separation in energy scales: very small $\Omega$ limit is interaction dominated and very large $\Omega$ limit is $\Omega$ dominated. In addition, for the very large $\Omega$ limit, the $\phi=0$ and $\pi$ cases are special because for these two cases there are $M$ different $\mathrm{U}(1)$ symmetries in the system corresponding to the conservation of particle numbers of each of the $\zeta$ flavors separately.

\section{A. $\Omega \ll \Omega_{c}$ case}

For this case, the ground state is the same for any value of $\phi$ since $\Omega_{\zeta} \approx 0$ implies no effect of $\phi$. It is made of $M$ baryons [35-38] which form a quasisuperfluid state described by an effective theory having central charge $c=1$. This is analogous to a Luther-Emery phase [49] with gapless "charge" excitations (which make up the unit central charge) and gapped flavor excitations. Within the canonical ensemble, $M$-particle excitations are of lowest energy.

\section{B. $\Omega \gg \Omega_{c}$ and $\phi=0$ case}

The off-diagonal elements of the $\mathbb{A}$ matrix generating the flavor-orbital coupling upon hopping are identically zero for $\phi=0$. The system for $\Omega \gg \Omega_{c}$, then, has populated states only in the $\zeta=1$ manifold as the Zeeman energy dominates over all the other energy scales. The system is a nearly free gas of fermions and corresponds to a flavor polarized "ferrometallic" state with lowest-energy one-particle excitations. The state can also be characterized by looking at the polarization of the particles in the original basis. E.g., for the $M=2$ case, the state is made of particles with the hyperfine states polarized along the " $x$ " direction in the original basis, i.e., the magnetic polarization $\mathcal{P} \equiv \sum_{i, \gamma, \gamma^{\prime}}\left\langle C_{i, \gamma}^{\dagger} \tau_{\gamma, \gamma^{\prime}}^{x} C_{i, \gamma^{\prime}}\right\rangle \neq 0$, where $\tau^{x}$ is the first Pauli matrix.

\section{C. $\Omega \gg \Omega_{c}$ and $\phi=\pi$ case}

The SD system has a special crisscross hopping structure for the $\phi=\pi$ case as shown in Fig. 2. As a result, the $\Omega \gg \Omega_{c}$ limit for the $\phi=\pi$ case is much more interesting than that of the $\phi=0$ case. Since in this limit only the $\zeta=1$ states make

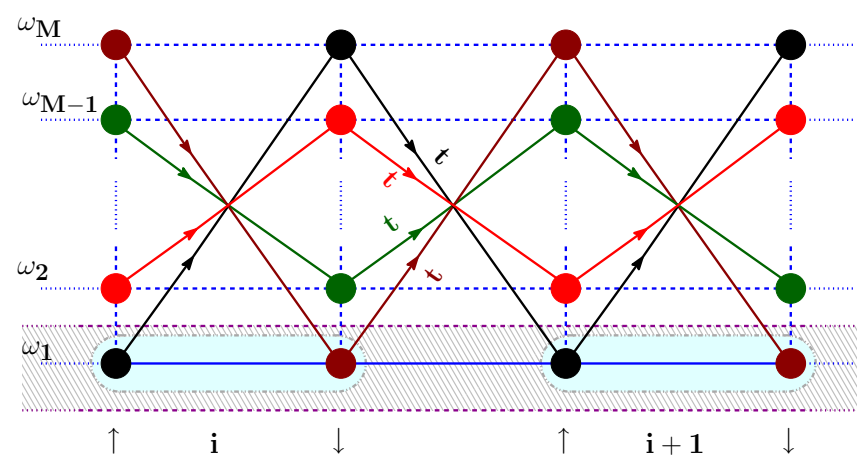

FIG. 2. Kinetic energy at $\phi=\pi$ : Schematic plot showing the crisscross hopping structure special to the $\phi=\pi$ case. For $\Omega \gg \Omega_{c}$, the low-energy sector is marked by the hatched box. In this limit, an effective theory can be constructed in the low-energy sector by considering two neighboring sites (shown by cyan boxes) to form a unit cell where the states on the odd (even) physical sites can be thought of as spin $\uparrow$ (spin $\downarrow$ ) states. The onsite energies of different $\zeta$ flavors are $\omega_{\zeta}$.

up the low-energy manifold, an effective theory for the system can be constructed with the Hilbert space made only of these states by projecting out other $\zeta$ flavor states. Then, the $\zeta=1$ states at the odd sites do not hybridize with those at the even sites - the system has an emergent $\mathrm{U}(1) \times \mathrm{U}(1)$ symmetry. Thus, without loss of generality, we can dub the odd site states as spin $\uparrow$ states and even site states as spin $\downarrow$ states. And, the system effectively is described by "spin- $\frac{1}{2}$ " fermions moving in a $1 \mathrm{D}$ chain.

We define new fermionic operators for this effective "spin$\frac{1}{2}$ " system by $b_{2 i-1,1}=a_{i, \uparrow}$ and $b_{2 i, 1}=a_{i, \downarrow}$. Here, $i$ labels the unit cells containing two neighboring sites labeled by $s$ ( $\uparrow$ and $\downarrow)$ as shown in Fig. 2. The effective Hamiltonian of the system is given by

$$
\begin{aligned}
\mathcal{H}_{\mathrm{eff}}= & -\sum_{i, s}\left[\left(a_{i+s, s}^{\dagger}+a_{i, s}^{\dagger}\right) \frac{t^{2}}{\left(2 \epsilon-U n_{i, \bar{s}}\right)} a_{i, s}\right. \\
& \left.+\left(a_{i, s}^{\dagger}+a_{i-s, s}^{\dagger}\right) \frac{t^{2}}{\left(2 \epsilon-U n_{i-s, \bar{s}}\right)} a_{i, s}\right]
\end{aligned}
$$

where $n_{i, s}=a_{i, s}^{\dagger} a_{i, s}$ is the number operator, $\epsilon=-\omega_{1}$, and we follow the notation of $s$ to be $+1(-1)$ for $\uparrow(\downarrow)$ particles and $\bar{s}=-s$. Then, using the identity

$$
\frac{1}{\left(2 \epsilon-U n_{i, s}\right)}=\frac{1}{2 \epsilon}\left[1+\frac{U n_{i, s}}{(2 \epsilon-U)}\right]
$$

since $n_{i, s}=0$ or 1 due to the fermionic nature of the particles, $\mathcal{H}_{\text {eff }}$ can be recast into the form

$$
\begin{aligned}
\mathcal{H}_{\text {eff }} & =\mathcal{H}_{1}+\mathcal{H}_{2}+\mathcal{H}_{3}, \\
\mathcal{H}_{1} & =-t_{\text {eff }} \sum_{i, s}\left(a_{i+s, s}^{\dagger} a_{i, s}+\text { H.c. }\right), \\
\mathcal{H}_{2} & =-U_{\text {eff }} \sum_{i, s}\left(n_{i, s} n_{i, \bar{s}}+n_{i+s, s} n_{i, \bar{s}}\right), \\
\mathcal{H}_{3} & =-U_{\text {eff }} \sum_{i, s}\left(a_{i+s, s}^{\dagger} a_{i, s}+\text { H.c. }\right) n_{i, \bar{s}},
\end{aligned}
$$


where $t_{\text {eff }}=\frac{t^{2}}{2 \epsilon}$ is the effective hopping amplitude and $U_{\text {eff }}=$ $\frac{t^{2} U}{2 \epsilon(2 \epsilon-U)}$ is the effective interaction. We note that to leading order there are two different kinds of interactions: a nonlocal interaction (given by $\mathcal{H}_{2}$ ) between particles at different physical sites of the optical lattice and a "correlated hopping" term (given by $\mathcal{H}_{3}$ ) which is also found in other contexts [50,51].

Now, we bosonize the effective Hamiltonian $\mathcal{H}_{\text {eff }}$ closely following Refs. $[44,45]$. The bosonized Hamiltonian, containing only the forward scattering terms, can be written in the following form:

$$
\begin{aligned}
\mathcal{H}_{b}^{0}= & \sum_{\nu=\rho, \sigma} v_{F}\left(1+\bar{g}_{4 v}\right) \sum_{q>0} q\left[\sum_{\eta=R, L} y_{q, \eta, \nu}^{\dagger} y_{q, \eta, \nu}\right. \\
& \left.+x_{\nu}\left(y_{q, R, \nu}^{\dagger} y_{q, L, \nu}^{\dagger}+\text { H.c. }\right)\right]+ \text { const. }
\end{aligned}
$$

Here, $v_{F}=2 t_{\text {eff }} \sin \left(k_{F}\right)$ is the Fermi velocity, $k_{F}$ is the Fermi momentum of each of the species $s, \eta$ labels the right $(R)$ and left $(L)$ movers, and $v$ labels the two different sectors' charge $(\rho)$ and $\operatorname{spin}(\sigma)$. The $y_{q, \eta, v}$ 's are the bosonic annihilation operators, $x_{v}=\frac{\bar{g}_{2 v}}{\left(1+\bar{g}_{4 v}\right)}$, and $\bar{g}_{m^{\prime} v}=\frac{g_{m^{\prime} v}}{v_{F}}$ with $m^{\prime}=2$ and 4 labeling the two types of forward scattering coupling constants. For this case,

$$
g_{m^{\prime} \rho}=-g_{m^{\prime} \sigma}=\frac{f\left(k_{F}\right)}{\pi},
$$

where $f\left(k_{F}\right)=V_{0}+V_{1}\left[1+2 \cos \left(k_{F}\right)\right]$ with $V_{0}=V_{1}=$ $-U_{\text {eff. }}$. Using the Bogoliubov transformation [45], the bosonized Hamiltonian in Eq. (9) can be diagonalized and in terms of the dual bosonic field operators $\phi_{v}(x)$ and $\theta_{v}(x)$ with $\Pi_{v}(x)=\partial_{x} \theta_{v}(x)$ it can be recast as

$$
\mathcal{H}_{b}^{0}=\sum_{v} \frac{u_{v}}{2} \int_{-\frac{L}{2}}^{\frac{L}{2}} d x\left[K_{v}: \Pi_{v}^{2}(x):+\frac{1}{K_{v}}:\left[\partial_{x} \phi_{v}(x)\right]^{2}:\right]
$$

The pair $\phi_{\nu}(x)$ and $\Pi_{v}(x)$ are canonically conjugate variables. Here, the Luttinger parameters $\left(K_{v}\right)$ are given by

$$
K_{v}=\sqrt{\frac{\left(1+\bar{g}_{4 v}-\bar{g}_{2 v}\right)}{\left(1+\bar{g}_{4 v}+\bar{g}_{2 v}\right)}}=\frac{1}{\sqrt{1-v \Lambda}},
$$

with $\Lambda=\frac{2 U \cot \left(\frac{k_{F}}{2}\right)}{\pi(2 \epsilon-U)}$ following the notation of $v$ to be $+1(-1)$ for particles in the $\rho(\sigma)$ sector. Their corresponding velocities are given by

$$
u_{v}=v_{F} \sqrt{\left(1+\bar{g}_{4 v}\right)^{2}-\left(\bar{g}_{2 v}\right)^{2}} .
$$

We note that in this large $\Omega$ limit under consideration, the parameter $\Lambda>0$ and hence $K_{\rho}>1$ and $K_{\sigma}<1$.

We now consider the effects of the backscattering terms [45] on the ground state of the Hamiltonian in Eq. (11) which contains only the forward scattering terms corresponding to $g_{2}$ and $g_{4}$. We are only interested in the physics of the system away from half filling, hence the important backscattering term is operational only in the spin sector $[44,45]$. Then, the bosonized Hamiltonian containing this spin backscattering term can be written as [using Eq. (11)]

$$
\mathcal{H}_{b}=\mathcal{H}_{b}^{0}+\frac{2 g_{1}}{(2 \pi \alpha)^{2}} \int d x \cos \left[\sqrt{8 \pi} \phi_{\sigma}(x)\right],
$$

with $\alpha$ being a microscopic length scale and $g_{1}$ being the spin backscattering coupling constant given by

$$
g_{1}=V_{0}+V_{1}\left[\cos \left(k_{F}\right)+\cos \left(2 k_{F}\right)+\cos \left(3 k_{F}\right)\right] .
$$

It is noted that for $U_{\text {eff }} \neq 0, g_{1} \neq 0$ and since $K_{\sigma}<1$ this term is relevant [44] and opens up gap in the spin sector. Thus, in this state, charge is gapless and the spin is gapped. The system has a dominant pair correlation $\Delta_{s}(r)$ which is defined as

$$
\Delta_{s}(r)=\left\langle\hat{D}_{j}^{\dagger} \hat{D}_{j+r}\right\rangle, \quad \hat{D}_{j}=a_{j, \uparrow} a_{j, \downarrow} \equiv b_{2 i-1,1} b_{2 i, 1} .
$$

It corresponds to a nonlocal correlation of squished pairs (nonlocal pairing in the optical lattice) $r$ distance away from each other. Closely following Ref. [45], we now obtain an explicit expression

$$
\Delta_{s}(r) \sim \frac{\beta_{1}}{r^{K_{\rho}}}-\frac{\beta_{2} \cos \left(2 k_{F} r\right)}{r^{\left(K_{\rho}+\frac{1}{K_{\rho}}\right)}},
$$

where $\beta_{1}$ and $\beta_{2}$ are positive parameters independent of $r$. Hence, the state is a Luther-Emery liquid (comprising nonlocal "spin- $\frac{1}{2}$ " singlets) with dominant pair correlations having unit central charge and lowest energy two-particle excitations. Also, it has $\mathcal{P}=0$ for $M=2$.

These analytical results allow us to arrive at some startling conclusions. Consider $\Omega \gg \Omega_{c}$; then, starting from a ferrometallic state at $\phi=0$, we can obtain a state with leading pair correlations by increasing $\phi$ to $\pi$. For any $M$, increasing the magnetic field through the plaquettes thus transforms a ferrometal to a quasisuperfluid of squished pairs.

\section{NUMERICAL RESULTS}

These intriguing analytical results clearly motivate a detailed numerical study of the many-body ground state of the system to understand this crossover from a ferrometal to a quasisuperfluid along with other possibilities which are not immediately evident from an analytical treatment. To this end, we perform large scale finite system numerical DMRG simulations for system sizes up to $L=160$ with open boundary conditions keeping up to 1000 matrix states.

We characterize different phases of the system by looking at several observables. First, we look for convenient local measures that provide signatures of the nature of the baryons (squished or otherwise) that make up the ground state. To this end, we define the following two operators: (1) local $p$-baryon creation operator $\hat{O}_{i, h}^{(p) \dagger}=b_{i, 1}^{\dagger} \ldots b_{i, p}^{\dagger}$ which creates a local $p$-baryon at site $i$ [schematically shown in Fig. 3(a)] and (2) squished $p$-baryon creation operator $\hat{O}_{i, s}^{(p) \dagger}=$ $b_{i, 1}^{\dagger} b_{i+1,1}^{\dagger} \ldots b_{i+p-1,1}^{\dagger}$ which creates a squished $p$-baryon of $\zeta=1$ flavor in the sites $i, \ldots,(i+p-1)$ [schematically shown in Fig. 3(b)]. We then calculate their average local correlations, called as local $p$-baryon amplitude

$$
f_{h}^{(p)}=1 / L \sum_{i}\left\langle\hat{O}_{i, h}^{(p) \dagger} \hat{O}_{i, h}^{(p)}\right\rangle
$$




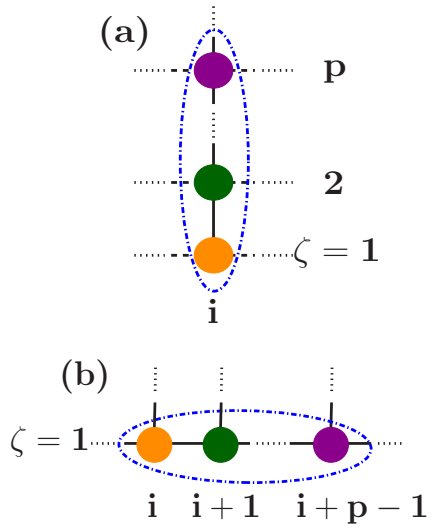

FIG. 3. Schematic view of (a) a local $p$-baryon at the site $i$ and (b) a squished $p$-baryon of $\zeta=1$ flavor in the neighboring sites of $i$.

and squished $p$-baryon amplitude

$$
f_{s}^{(p)}=1 / L \sum_{i}\left\langle\hat{O}_{i, s}^{(p) \dagger} \hat{O}_{i, s}^{(p)}\right\rangle,
$$

where $p$ can take values $2,3, \ldots, M$. A state consisting of the usual $M$-baryons will have a dominant value of local $M$-baryon amplitude $\left(f_{h}^{(M)}\right)$ together with a vanishing value of squished $M$-baryon amplitude $\left(f_{s}^{(M)}\right)$ but in contrast a state with squished baryons will have the opposite. These two quantities together, therefore, provide a measure to distinguish between the usual and the squished baryon states. We also look at long-distance correlation functions: (1) "nonlocal squished pair" correlation function $\Delta_{s}(r)=\left\langle\hat{O}_{i, s}^{(2) \dagger} \hat{O}_{i+r, s}^{(2)}\right\rangle$ with $r$ even, which is equivalent to Eq. (16) and captures the squishing effect, and (2) "local-pair" correlation function $\Delta_{h}(r)=\left\langle\hat{O}_{i, h}^{(2) \dagger} \hat{O}_{i+r, h}^{(2)}\right\rangle$ which corresponds to a pair-correlation function of usual dimers.

Further, we compute the lowest $m$-particle excitation energy of the ground state within the canonical ensemble defined by

$$
\Delta E_{m}=\frac{E_{0}(N+m, L)+E_{0}(N-m, L)-2 E_{0}(N, L)}{2 m},
$$

where $E_{0}(N, L)$ is the ground-state energy of the SD system with $L$ optical lattice sites and $N$ particles. One of the most important tools, however, to characterize various gapless phases with different central charge $(c)$ is the von-Neumann block-entanglement entropy $S_{\mathrm{vN}}=-\operatorname{tr}\left(\rho_{l} \ln \rho_{l}\right)$, where $\rho_{l}$ is the reduced density matrix of a subsystem of length $l$ embedded in a chain of finite length $L$. For conformally invariant gapless states, the scaling of the entanglement entropy can be related to the central charge of the system $[52,53]$. In particular, for different subsystem lengths of 1D systems with open boundaries, $S_{\mathrm{vN}}(l)$ is described by the Calabrese-Cardy-formula [54]:

$$
S_{\mathrm{vN}}(l)=\frac{c}{6} \ln \left[\frac{L}{\pi} \sin \left(\frac{\pi}{L} l\right)\right]+\cdots .
$$

We extract the central charge (c) of the SD system by fitting the numerically computed $S_{\mathrm{vN}}$ to this Calabrese-Cardy formula.

We begin the discussion of the numerical results in the next section by first comparing them with the analytical

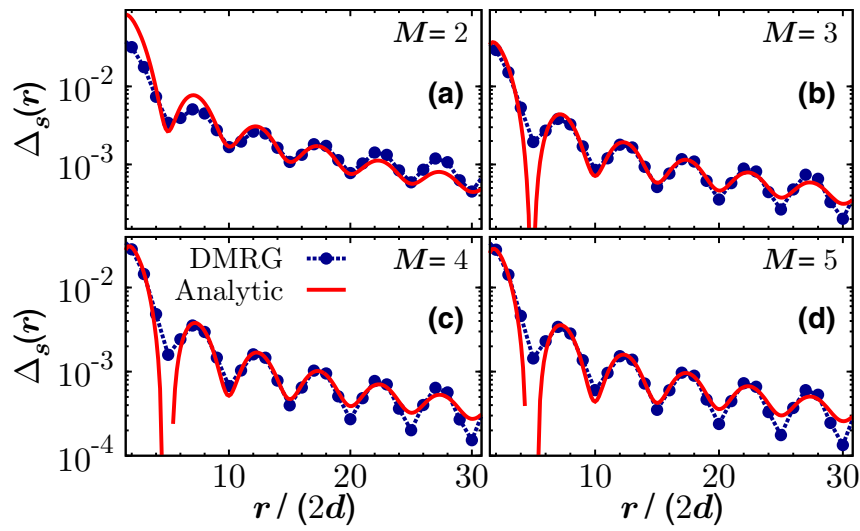

FIG. 4. Squished pair-correlation functions: Comparison of DMRG and field theoretic results of the algebraic decay of the nonlocal pair correlation $\Delta_{s}(r)$ for $\pi$ flux with $t / U=0.5, \Omega / U=4$, $L=160$, and $N=32$. Panels (a), (b), (c), and (d) show the $M=2$, 3,4 , and 5 cases, respectively.

results obtained from the effective field theory described in the previous section for the limiting case $\Omega \gg \Omega_{c}$ with $\phi=\pi$. Then, we move away from the analytically predictable regimes, in order to map out the full phase diagram considering the $M=2$ and 3 cases as examples.

\section{A. Analytics versus DMRG for $\phi=\pi$}

In Fig. 4, we show the comparison between the bosonization result [Eq. (17)] and the DMRG result of $\Delta_{s}(r)$ for the $\pi$-flux case in the limit of $\Omega \gg \Omega_{c}$ for different $M$ SD systems. The Fermi momentum $k_{F}$ used in Eq. (17) is given by $k_{F}=\frac{\pi}{2} M n$ and the DMRG data are fitted by the bosonization result using $\beta_{1}$ and $\beta_{2}$ as fitting parameters. As is evident, the numerical results are in excellent agreement with those predicted by the field theory for all values of $M$ considered, confirming the analytical prediction of the formation of the "squished baryon" quasicondensate in this limit.

To further elucidate the algebraic decay of the squished pair-correlation function $\Delta_{s}(r)$ and to verify that it is indeed the dominant correlation of the system for this case, we plot it in log-log scale and compare it with another relevant correlation function, the local pair-correlation function $\Delta_{h}(r)$ for the $M=$ 2 case as an example in Fig. 5. We note from this figure that $\Delta_{s}(r)$ is much larger than $\Delta_{h}(r)$ and $\Delta_{s}(r)$ indeed decays algebraically.

In the following two sections, we give detailed description of the many-body phase diagram obtained numerically considering the $M=2$ and 3 cases as examples.

\section{B. DMRG results for $M=2$}

In this section, we discuss the DMRG results for the $M=2$ system (see Refs. [55-58] for studies on similar two-leg ladders but with repulsive interaction) in the $\Omega / U-\phi$ parameter space for a fixed finite $t$ summarized in Fig. 6. We show the many-body ground-state phase diagram of the system in Figs. 6(a), 6(b), and 6(c) in the $\Omega / U-\phi$ plane considering the squished two-baryon amplitude $\left(f_{s}^{(2)}\right)$, the local two-baryon amplitude $\left(f_{h}^{(2)}\right)$, and the central charge $(c)$, 


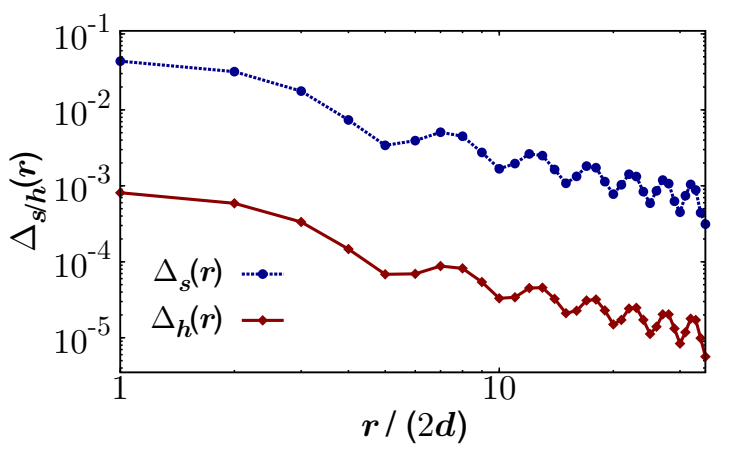

FIG. 5. Comparison of the DMRG results of the correlation functions of squished pairs $\Delta_{s}(r)$ and usual dimers $\Delta_{h}(r)$ of the $M=2 \mathrm{SD}$ system for $\pi$ flux with $t / U=0.5, \Omega / U=4, L=160$, and $N=32$ (lines are a guide to the eye).

respectively. Taken together, they characterize the different phases shown in these figures. We also show the variation of the lowest-energy single- and two-particle excitations for three different values of $\phi=0.05 \pi, 0.2 \pi$, and $\pi$ as a function of $\Omega / U$ in Figs. 6(d), 6(e), and 6(f), respectively, and for large $\Omega / U=0.8\left(>\Omega_{c}\right)$ as a function of $\phi / \pi$ in Fig. $6(\mathrm{~g})$.

We start the discussion by first considering the $\phi \approx 0$ limit. We note that in this limit, when $\Omega \ll \Omega_{c}\left(\Omega_{c} \leqslant \Omega_{c}^{0}=U / 2\right)$, $f_{s}^{(2)}$ is small while $f_{h}^{(2)}$ is large, indicating the presence of the usual two-baryons - the state thus is a two-baryon quasisuperfluid (2-BF) [see Figs. 6(a) and 6(b)]. The signature of Luther-Emery physics in this state is further corroborated by (i) the unit value of the central charge $c=1$ [Fig. 6(c)] and (ii) the lowest-energy excitation being a two-particle one [Fig. 6(d)], both of which are due to the two-baryons. But when $\Omega \gg \Omega_{c}$, we note that both the amplitudes $f_{s}^{(2)}, f_{h}^{(2)} \approx 0$. Thus there are no pairing correlations in the system. Further, the central charge $c=1$ but now it is due to the single-particle degrees of freedom, which is also seen in Fig. 6(d) by noting the lowest-energy excitations to be the one-particle ones. All of these signatures confirm the expected flavor polarized "free" gas of $\zeta=1$ fermions-the ferrometallic (FM) state. In this $\phi \approx 0$ limit, the 2-BF phase and the FM phase are separated by an intervening Fulde-Ferrell-Larkin-Ovchnikov (FFLO) phase with $c=2$ at an "intermediate" value of $\Omega$. The existence of this phase can be qualitatively understood by noting that this SD system with $\phi=0$ is exactly equivalent to the Hubbard model of spin- $\frac{1}{2}$ fermions in an external Zeeman field and this model has an extended FFLO regime for "intermediate" values of the magnetic field [59]. Thus, the SD system also has an FFLO region for $\phi=0$ and for small $\phi \approx 0$; its precise extent is characterized by looking at the central charge $c=2$, excitation spectrum, and imbalance between the total average populations of the two flavors. On a qualitative level, the FFLO phase can be understood as a partially paired phase with both an unpaired atomic liquid component and a paired superfluid. With this argument, the observed central charge $c=2=1+1$ becomes clear. We also note that the partially paired nature of the FFLO phase is manifested in the irregular oscillatory behaviors of the single- and two-particle excitations as seen in Fig. 6(d).
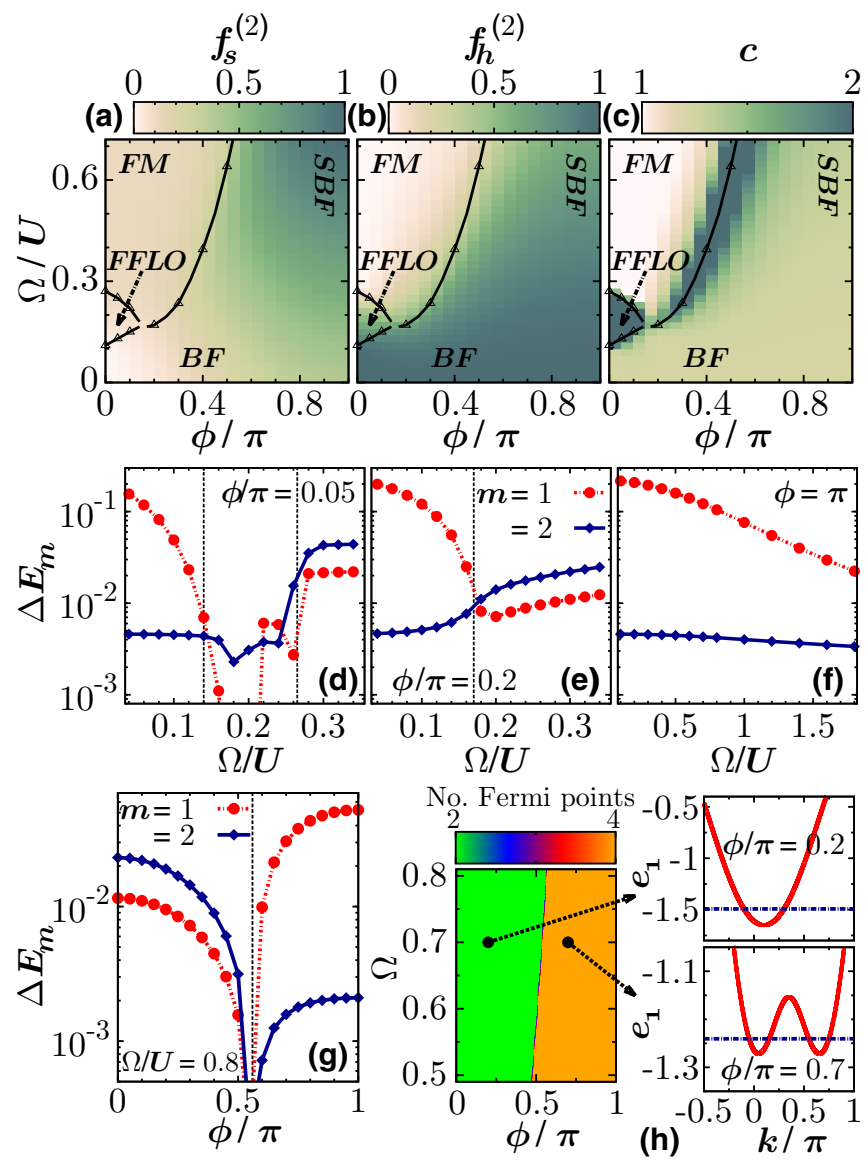

FIG. 6. $M=2$ results: $t / U=0.5, n=0.1$. Phase diagrams in the $\Omega-\phi$ plane corresponding to (a) normalized $f_{s}^{(2)}$, (b) normalized $f_{h}^{(2)}$, and (c) central charge $c$ (DMRG data for $L=80$ sites). $f_{s}^{(2)}$ and $f_{h}^{(2)}$ are normalized by their maximum values in the $\Omega-\phi$ plane shown here $\left(\operatorname{Max}\left[f_{s}^{(2)}\right]=0.0428\right.$ and $\left.\operatorname{Max}\left[f_{h}^{(2)}\right]=0.0466\right)$. Symbols depict estimates of transition points by DMRG while the solid lines are guides to the eye. Excitation spectra are shown, along different cut directions of the phase diagrams, first in (d), (e), and (f) vs $\Omega$ for $\phi / \pi=0.05,0.2$, and 1 , respectively, and then in (g) vs $\phi$ for fixed $\Omega / U=0.8(L=80)$. Dashed lines show the transition points between different phases seen in the phase diagrams. In the large $\Omega>$ $\Omega_{c}$ limit, variation of the number of Fermi points of the corresponding noninteracting system in the $\Omega-\phi$ plane is shown in the left panel of (h). Its right panels show the structure of the Fermi surface with the blue lines showing the chemical potential in the first band $\left[e_{1}(k)\right]$ for the chosen filling.

Moving to an "intermediate" regime of $\phi$, we see from the phase diagrams that 2-BF and FM phases occur in the expected regimes without any intervening FFLO phase. This is also seen from Fig. 6(e) considering a particular value of $\phi$ in this regime. We note that a rather sharp transition occurs from the $2-\mathrm{BF}$ to the $\mathrm{FM}$ phase at $\Omega / U \approx 0.17$. Now, we move onto the more interesting case $\phi=\pi$. For this case, the 2 -BF state for $\Omega \ll \Omega_{c}$ smoothly crosses over to the squished baryon fluid (SBF) at large $\Omega$ (confirming the analytical prediction) - throughout this process the central charge remains unity although the degrees of freedom making up the central charge are different for the two states. And, the 
lowest-energy excitations are two-particle ones for all values of $\Omega$ as is seen from Fig. 6(f).

The most remarkable aspect of the phase diagram occurs in the limit of $\Omega>\Omega_{c}$. In this limit, it is evident from the excitation spectrum shown in Fig. $6(\mathrm{~g})$ that with changing $\phi$ the FM state at $\phi=0$ switches over to the SBF state at $\phi=\pi$ precisely as anticipated from the analytical theory. This change is intervened by a critical point at $\phi=\phi_{t}$ (e.g., $\phi_{t} \approx 0.56 \pi$ for $\Omega / U=0.8$ ). We argue that a change in the topology of the Fermi surface, i.e., a Lifshitz transition [60,61], of noninteracting fermions underlies this critical point as illustrated in Fig. 6(h). In the left panel of Fig. 6(h), we show the variation of the number of Fermi points of the noninteracting Fermi surface in the large $\Omega\left(>\Omega_{c}\right)$ limit as a function of $\phi$. This is obtained by a construction shown in the right panels of Fig. 6(h) by suitably fixing the chemical potential of the system for the particular density. It is now clear that the locus of transition points from FM to SBF can be understood as that of a Lifshitz transition from a Fermi surface having two Fermi points to that having four Fermi points occurring in the noninteracting SD system dressed by interactions. Interestingly, a detailed numerical analysis of this transition described in Appendix A indicates that there is a finite parity order in the SBF phase. Furthermore, the different phases described here can also be characterized by their crucial signatures in the experimentally measurable chiral currents $[16,17]$ as discussed in detail in Appendix C.

\section{DMRG results for $M=3$}

We summarize the main results of the $M=3$ case in Fig. 7. The many-body phase diagrams characterized by the following quantities-squished three-baryon amplitude $\left(f_{s}^{(3)}\right)$, local three-baryon amplitude $\left(f_{h}^{(3)}\right)$, and central charge (c) -are shown in Figs. 7(a), 7(b), and 7(c), respectively. The lowest-energy excitation spectra of the systems for different parameters are shown in Figs. 7(d)-7(f). In a similar spirit of the $M=2$ case discussed in the previous section, we note that for $\phi \approx 0$ and $\Omega \ll \Omega_{c}\left(\Omega_{c} \leqslant \Omega_{c}^{0}=U / \sqrt{2}\right)$ the state is a quasisuperfluid of three-baryons (which are fermions). It is abbreviated as the 3-BF state and its presence is indicated by a large $f_{h}^{(3)}$ along with a vanishing $f_{s}^{(3)}$ as shown in Figs. 7(a) and 7(b). In fact, this state with $c=1$ (due to the three-baryons) prevails for all $\phi$ when $\Omega \ll \Omega_{c}$ shown in Fig. 7(c). For $\phi \approx 0$, we obtain the flavor polarized FM state when $\Omega \gg \Omega_{c}$ and it is separated from the 3-BF state by an intervening FFLO phase (with $c>1$ ) similar to the $M=2$ case. This is also clear from the excitation spectrum shown in Fig. 7(d) for $\phi=0$. From this figure, we note that as expected the lowest-energy excitation in the 3-BF state is of three-particle type whereas that in the FM phase is of one-particle type.

It is evident from the phase diagrams that this case has qualitatively different phases than the $M=2$ case and is much richer. Concentrating on the $\Omega \gg \Omega_{c}$ limit, we note that as a function of $\phi$ the FM state of the system at small $\phi$ becomes an SBF state made of squished two-baryons very close to the $\pi$ flux as predicted by the analytical theory. But, there is also an imbalanced phase which appears just before the appearance of the SBF state. The nature of the different phases in this limit can be further understood by looking at the excitation spectrum
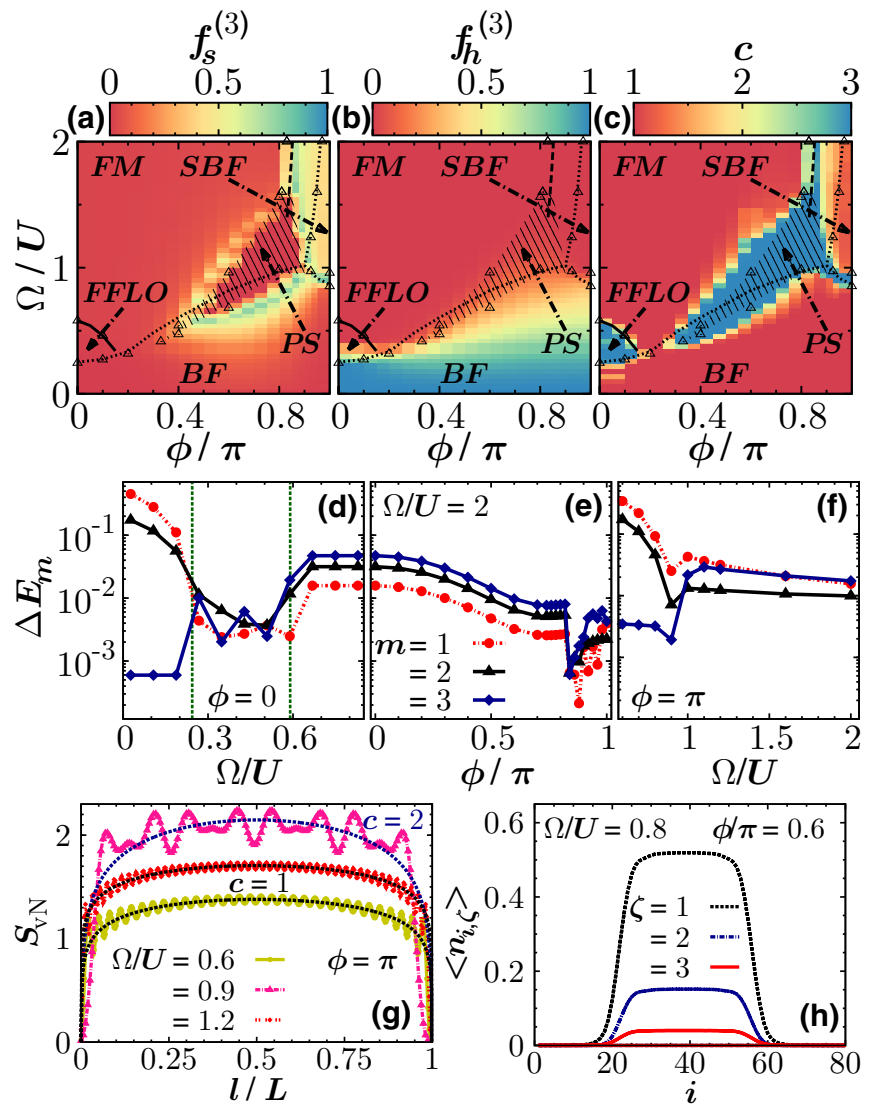

FIG. 7. $M=3$ results: $t / U=0.5$ and $n=0.1$. Phase diagrams corresponding to (a) normalized $f_{s}^{(3)}$, (b) normalized $f_{h}^{(3)}$ and (c) central charge $c$, respectively $(L=40) . f_{s}^{(3)}$ and $f_{h}^{(3)}$ are normalized by their maximum values in the $\Omega-\phi$ plane shown here. The maximum value of $f_{h}^{(3)}$ is $\operatorname{Max}\left[f_{h}^{(3)}\right]=0.0566$ and the maximum value of $f_{s}^{(3)}$, calculated by "masking" the phase separation (PS) region shown by the hatched lines and setting $f_{s}^{(3)}$ to be zero in this region, is $\operatorname{Max}\left[f_{s}^{(3)}\right]=0.0226$. Symbols, estimates of transition points by DMRG; solid lines, guides to the eye; dotted lines, numerical estimates of bound-state transitions in the dilute limit; and dashed lines, Lifshitz transitions (also discussed in Appendix A). (d)-(f) Excitation spectra along different cuts of the phase diagrams $(L=80$; green dotted lines, extent of the FFLO region). (g) $S_{\mathrm{vN}}$ of a subsystem of size $l$ with fits to the Calabrese-Cardy formula [54] for $L=160$ and $\phi=\pi$. (h) Variations of onsite populations $\left\langle n_{i, \zeta}\right\rangle$ in the phase separation (PS) regime $(L=80)$, and clustering of particles near the central site is seen.

shown in Fig. 7(e). The lowest-energy one-particle excitations of the FM state become two-particle type in the SBF state as a function of $\phi$, as expected. Further, similar to the $M=2$ case the transition to the SBF state in the limit $\Omega \gtrsim \Omega_{c}$ can be understood by looking at the Lifshitz transitions occurring in the noninteracting Fermi surface. As shown in Fig. 8, there are interesting Lifshitz transitions occurring in the noninteracting Fermi surface in this limit. And, the transition to the SBF state is associated with the Lifshitz transition of the Fermi surface having two Fermi points to that having four Fermi points. There are also other interesting Lifshitz transitions from two to six to four Fermi points as a function of $\phi$ at fixed $\Omega$ but the precise characterization of the transitions of different 


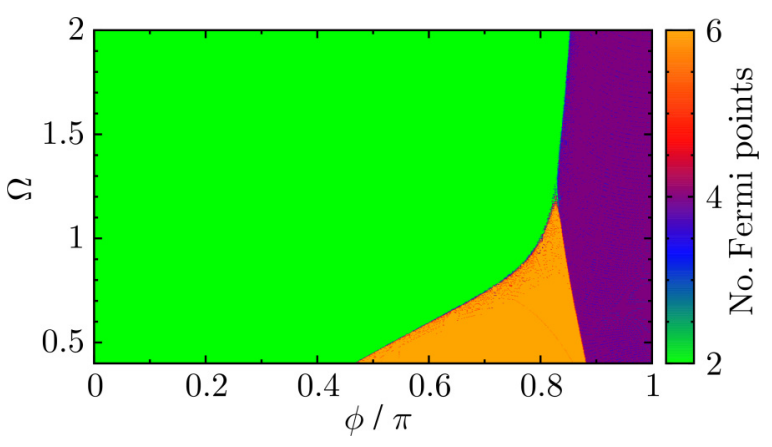

FIG. 8. Lifshitz transitions occurring in the $\Omega-\phi$ plane for the noninteracting $M=3 \mathrm{SD}$ system with $t=0.5$ and $n=0.1$ are shown here by looking at the number of Fermi points in the noninteracting Fermi surface with the given density.

phases using these Lifshitz transitions is complicated by the intervening extended phase separation (PS) region (described below) shown by the hatched lines in the phase diagrams.

Matters take a dramatic turn near $\Omega \approx \Omega_{c}$ and $0.4 \pi \leqslant \phi \leqslant$ $0.7 \pi$-the hatched region in Figs. 7(a)-7(c). In this regime, the system displays PS as is seen from Fig. 7(h). In this figure, the variation of the average onsite populations $\left\langle n_{i . \zeta}\right\rangle$ of different flavors in the phase separation regime is shown. We note that the central region is of the highest density, signaling clear indication of phase separation with tendency of clustering of particles near the central site. The propensity of phase separation can be further understood by noting the increased "flatness" of single-particle bands in this regime and looking at the equation of state of the system as discussed in Appendix B. It is indeed noteworthy that this system can be used to study the physics of quantum phase separation in a multiflavor fermionic setup.

The physics at $\phi=\pi$, for this $M=3$ case, has some more interesting aspects than that of the $M=2$ case. Increasing $\Omega$ from $\Omega \ll \Omega_{c}$ for $\phi=\pi$ results in an interesting transition from the 3-BF to the SBF state having three-particle and two-particle lowest-energy excitations, respectively, as shown in Fig. 7(f). This transition is also intervened by a "small" intermediate regime where the three-baryons are destabilized in favor of the squished two-baryons. As explained in Appendix A, there are strong finite-size effects in the estimation of central charge especially in imbalanced regimes. To reduce this effect, we consider a larger system size $L=160$ for the $\pi$ flux case using the enhanced symmetries special to this case. The result is shown in Fig. 7(g). We note that both the 3 -BF and the SBF states have $c=1$ while in the intermediate regime the fitting of the $S_{\mathrm{vN}}(l)$ with the Calabrese-Cardy formula is more compatible with $c=2$. The fact that $c=2$ in this regime suggests that the low-energy physics has two types of excitations and constructing a field theoretic description of the corresponding state will be an interesting future direction. The transitions between different phases can also be identified by looking at the variations of chiral currents as discussed in Appendix C.

The picture just described can be further confirmed by looking at the variations of the average total population $N_{\zeta}$ of different $\zeta$ flavors defined as $N_{\zeta}=\sum_{i}\left\langle n_{i, \zeta}\right\rangle$. This is shown in Fig. 9. From the panel Fig. 9(a), we note that at $\phi=0$
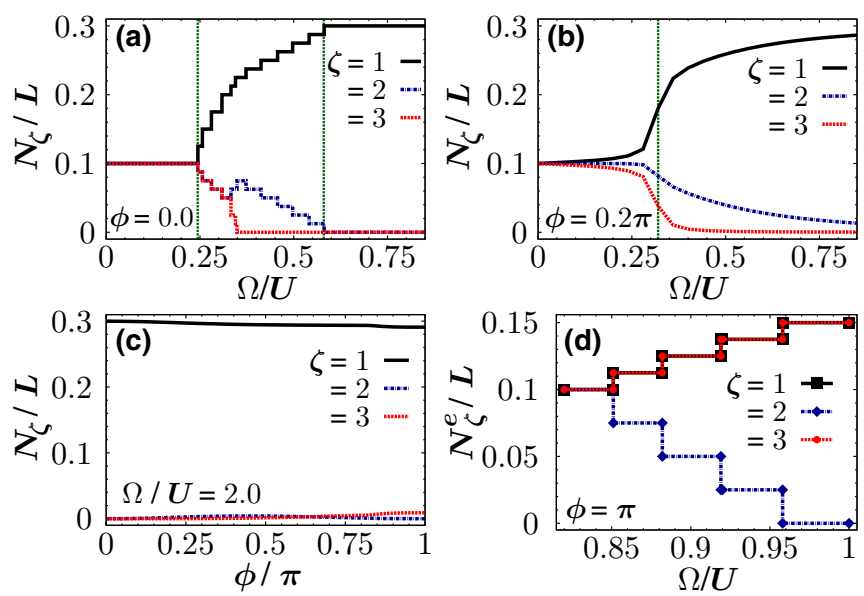

FIG. 9. Population $(M=3)$ : The variations of the total average populations $\left(N_{\zeta}\right)$ of different $\zeta$ flavors are shown for the $M=3$ case with $t / U=0.5, n=0.1$, and $L=80$. (a) Variations of $N_{\zeta}$ as a function of $\Omega$ for the $\phi=0$ case with the green lines showing the extent of the FFLO region. Similarly, in (b) we show the same for the $\phi=0.2 \pi$ case with the green line showing the transition point from the 3-BF state to the FM state, while in (c) we show the variations of $N_{\zeta}$ as a function of $\phi$ in the $\Omega \gg \Omega_{c}$ with $\Omega / U=2$. Finally, for the special case $\phi=\pi$, we show the variations of the effective populations $N_{\zeta}^{e}$ as a function of $\Omega$ in (d).

for small $\Omega$, the populations of all the $\zeta$ flavors are equal as expected for a $3-\mathrm{BF}$ state. With increasing $\Omega$, there is a regime of $\Omega$ over which populations of different flavors are unequal but nonzero, i.e., there is imbalance between different flavors. This regime corresponds to the FFLO state. For very large $\Omega$, the state becomes an FM state when all the states are of $\zeta=1$ flavor. The plateaus in the FFLO regime are due to the three separate $U(1)$ symmetries corresponding to the particle number conservation of the individual flavors special to this case and finite system size. In contrast, for the $\phi=0.2 \pi$ case shown in Fig. 9(b), there is a direct transition from the 3-BF state to the FM state without any intervening FFLO phase as also seen in the phase diagrams Figs. 7(a)-7(c). We expect the nonsharpness of the present plot to be related to finite-size effects. Further, for the interesting limit of $\Omega \gg \Omega_{c}$ considered in Fig. 9(c), we note that $N_{1}$ is always the largest whereas $N_{2} \approx N_{3} \approx 0$.

Interestingly, for the $\pi$-flux case we can define effective populations $N_{\zeta}^{e}$ of different $\zeta$ flavors for this $M=3$ case as

$$
\begin{gathered}
N_{1}^{e}=\sum_{i=1}^{L / 2}\left(\left\langle n_{2 i-1,1}\right\rangle+\left\langle n_{2 i, 3}\right\rangle\right), \\
N_{2}^{e}=N_{2}, \\
N_{3}^{e}=\sum_{i=1}^{L / 2}\left(\left\langle n_{2 i-1,3}\right\rangle+\left\langle n_{2 i, 1}\right\rangle\right) .
\end{gathered}
$$

Variation of $N_{\zeta}^{e}$ as a function of $\Omega$ is shown in Fig. 9(d). It is noted that at small $\Omega$ all the effective populations are equal but at large $\Omega\left(>\Omega_{c}\right) N_{1}^{e}=N_{3}^{e}$ while $N_{2}^{e}=0$. These two states correspond to the 3-BF state and the SBF state, respectively. The effective $\mathrm{U}(1) \times \mathrm{U}(1)$ symmetry is thus evident in the 
SBF state. We also note that for intermediate $\Omega$, there is an imbalanced regime over which $N_{2}^{e} \neq 0$ but $N_{2}^{e} \neq N_{1}^{e}=N_{3}^{e}$ as pointed out earlier.

\section{SUMMARY AND OUTLOOK}

In summary, we have presented an extensive study of the rich physics of a fermionic SD system with $\mathrm{SU}(M)$ symmetric attractive interaction. Moving to a flavor basis, in which the multiflavor fermions experience a competition between an $\mathrm{SU}(M)$ non-Abelian gauge field and a Zeeman field, we analyze the many-body phase diagram of the system using both analytical and numerical techniques. It was shown that an effective description in terms of "spin- $\frac{1}{2}$ " fermions experiencing long-ranged interaction and correlated hopping is possible for the system with $\pi$ flux and large Raman coupling strength. We show that the system in this case becomes a Luther-Emery liquid with algebraically decaying pair correlation by using field theory and comparing with DMRG results. Further, we uncover the rich ground-state phase diagram of the system with several interesting phases concentrating in particular on the $M=2$ and 3 cases. There are several interesting transitions or crossovers between the phases, such as the fact that a flavor polarized ferrometallic state can turn into a quasisuperfluid state with algebraically decaying pair correlation with changing flux. In addition, there is an interesting regime in the phase diagram for $M=3$ where there is phase separation.

Experimentally, SD systems having up to $M \leqslant 6$ can be realized using the recently studied $\mathrm{Yb}^{173}$ system [17] and orbital Feshbach resonance [25-27] in this system can produce the $\mathrm{SU}(M)$ symmetric interaction. Also, the SU(3) symmetric $M=3 \mathrm{SD}$ system can be realized using nuclear spin- $1 \mathrm{Li}^{6}$ atoms with a large negative triplet scattering length [62]. Different phases in the many-body phase diagram can be characterized by using familiar band mapping techniques [63-66] along with photoassociation spectroscopy [67] (for example, a state with local baryons will produce a "large" photoassociation signal whereas a state consisting of nonlocal baryons will give rise to a "small" photoassociation signal). Also, the chiral currents, measured in the experiments $[16,17]$, point towards interesting additional structures of different phases and further characterize them (discussed further in Appendix C).
In view of the significant recent advances on the experimental front, we expect the predictions of the unusual physical phenomena presented here will trigger a whole line of research aiming at realizing exotic states of synthetic quantum matter including connections to quantum chromodynamics [68].

\section{ACKNOWLEDGMENTS}

S.G. acknowledges the support of the Research Training Group 1729 and Project No. SA 1031/10-1 of the German Research Foundation. S.G. also thanks Temo Vekua, Luis Santos, and Leonardo Mazza for enlightening discussions and acknowledges Leibniz University of Hannover, Germany for computing resources. T.M. would like to acknowledge support by a start-up research grant from the Indian Institute of Technology, Guwahati, India. V.B.S. is grateful to DST, India and DAE, India (SRC grant) for generous support.

\section{APPENDIX A: FURTHER NUMERICAL ANALYSIS}

In order to obtain a full picture of the nature of the ground state in the dilute limit $(n \ll 1 / M)$, we evaluate the energies of multiparticle bound states of the SD system numerically. We compute the energies of two-, three-, and four-particle states in an empty lattice by means of DMRG simulations for the $M=2,3$, and 4 cases, respectively. When compared with the corresponding single-particle energies, they allow us to estimate the positions of different transitions. In Fig. 10, we depict the transition lines as well as the Lifshitz transition line which occurs in the large $\Omega$ limit. The estimates of the transitions are shown as different lines in the phase diagrams shown in Figs. 6 and 7. They compare well with the corresponding numerical estimates from excitation spectra.

We also compute for the $M=2$ case the averaged entanglement entropy $\mathrm{S}_{\mathrm{vN}}^{\mathrm{av}}$ over all bipartitions $(l)$ in the range $[L / 4,3 L / 4]$ reducing the finite-size effects and it increases in the FFLO regime as shown in the inset of Fig. 11(a). The central charge $(c)$ is estimated directly by fitting the numerical results with Eq. (21) as shown, for example, in Fig. 11(b). However, for finite systems with open boundary conditions and in particular for low fillings, strong oscillatory terms complicate this analysis. Hence, the central charge in the intermediate imbalanced phase is overestimated due to the finite system size effects. This can be seen from Fig. 11(b) for the $M=3$ case with $\pi$ flux as an example.
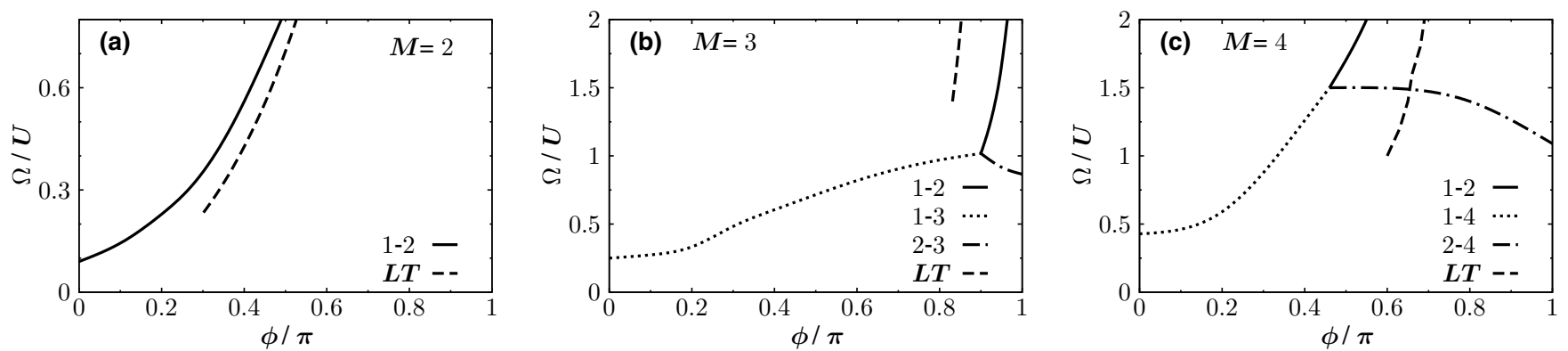

FIG. 10. Phase diagram in the dilute limit $n \approx 0$, for (a) $M=2$, (b) $M=3$, and (c) $M=4$. Solid, dotted, and dash-dotted lines indicate various crossings between different few-particle bound-state energies to be the lowest energy for the few-particle system. For the sake of completeness, we add the Lifshitz-transition lines (dashed lines) in the large $\Omega$ limit. 

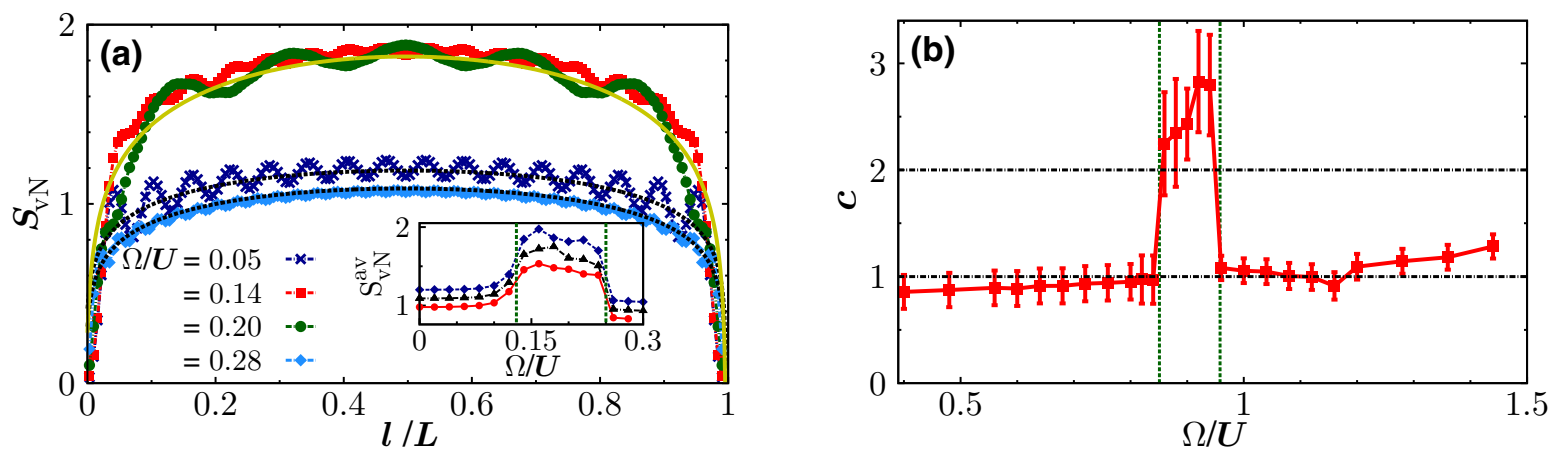

FIG. 11. (a) Entanglement entropy $S_{\mathrm{vN}}$ as a function of the subsystem size $l$ for the $M=2$ case with $\phi=0.05 \pi, t / U=0.5$, and several values of $\Omega$. The black dotted lines are fits to the Calabrese-Cardy-formula [Eq. (21)] with $c=1$ in the $2-\mathrm{BF}(\Omega / U=0.05)$ and FM $(\Omega / U=0.28)$ phases depending on the data, while the solid yellow line is the same with $c=2$ in the FFLO region $(\Omega / U=0.14$ and 0.2$)$. The inset shows the average entanglement entropy $S_{\mathrm{vN}}^{\mathrm{av}}$ as a function of $\Omega / U$ for system sizes $L=40,80$, and 160 from bottom to top and the green dotted lines denote the extent of the FFLO region as determined by the excitation spectra defined in Eq. (20). (b) The estimated central charge (c) using similar procedure as (a) for the $M=3$ case with $\phi=\pi$ and $t / U=0.5$ as a function of $\Omega / U$ ( $L=80$ ). Corresponding excitation spectra are shown in Fig. 7(f). While for the $3-\operatorname{BF}(\Omega / U \lesssim 0.85)$ and the $\operatorname{SBF}(\Omega / U \gtrsim 0.95)$ phases we observe $c=1$, the fitting result for the intermediate imbalanced phase may be consistent with $c=2$. The green dotted lines again denote the extent of the imbalanced region as determined by the excitation spectra.

Finally, we present details of the transition between the FM and SBF phases for the $M=2$ case in the large $\Omega$ limit as a function of $\phi$. Contrary to the $M=3$ case, we observe a single critical point between the two phases without crossing to an intermediate phase. In Fig. 12, we present data for a cut that is the same as in Fig. 6(g) (showing the corresponding excitation spectra) for $\Omega / U=0.8$. This gives strong indications of the presence of a "critical" point. Indeed, similar critical points between Luttinger liquids of bound and unbound pairs have been discussed, for example, in spin systems [69] and bosonic systems [70,71].

In Fig. 12(a), we show the scaling of the fidelity susceptibility [72] defined as

$$
\chi_{F}(\phi)=\lim _{\delta \phi \rightarrow 0} \frac{-2 \ln \left|\left\langle\Psi_{0}(\phi) \mid \Psi_{0}(\phi+\delta \phi)\right\rangle\right|}{(\delta \phi)^{2}}
$$

with $\left|\Psi_{0}\right\rangle$ being the ground-state wave function. As the system size increases, $\chi_{F} / L^{2}$ develops a distinct divergent single peak and the peak height $\left(\chi_{F}^{\max } / L^{2}\right)$ scales linearly with respect to its wings $[73,74]$. Note that since we are calculating the

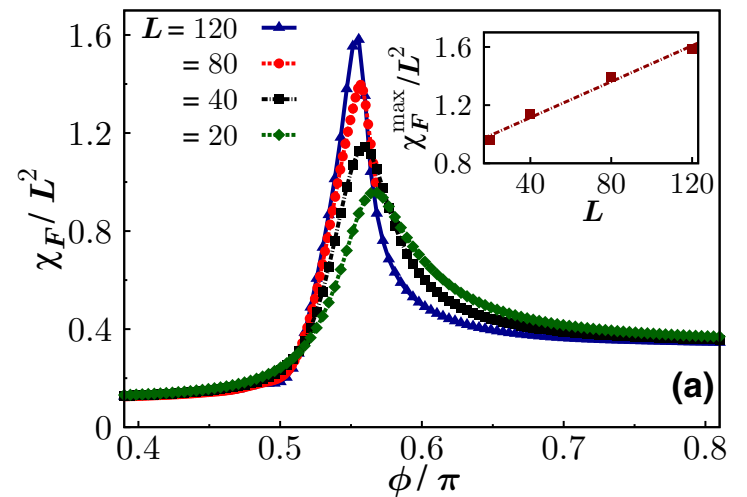

fidelity susceptibility with respect to the flux one generally observes a quadratic scaling of the fidelity susceptibility with the system size $\chi_{F} \sim L^{2}[73,74]$. Equivalently, for a crossing of the transition at fixed flux, we observe that $\chi_{F} \sim L$ within the FM and SBF phases and $\chi_{F} \sim L^{2}$ for the transition.

An "order parameter" of this transition can be constructed and it is given by the parity order

$$
\mathcal{O}_{P}^{2}(i, j)=\mathrm{e}^{i \sum_{i<k<j} \sum_{\zeta} \pi n_{k, \zeta}} .
$$

In Fig. 12(b), we plot the averaged parity order $\mathcal{O}_{P}^{2}=$ $\frac{2}{L} \sum_{L / 4<j<3 L / 4}\left\langle\mathcal{O}_{P}^{2}(L / 4, j)\right\rangle$ as a function of the flux for several system sizes. The parity order vanishes in the FM phase $\mathcal{O}_{P}^{2} \rightarrow 0$ while it becomes finite in the SBF phase and there is an interesting scaling as illustrated in the inset of Fig. 12(b).

\section{APPENDIX B: PHASE SEPARATION}

The propensity of phase separation for the $M=3$ case is further analyzed in this section. In Fig. 13(a), we show the equation of state $n(\mu)$, where $\mu$ is the chemical potential of the

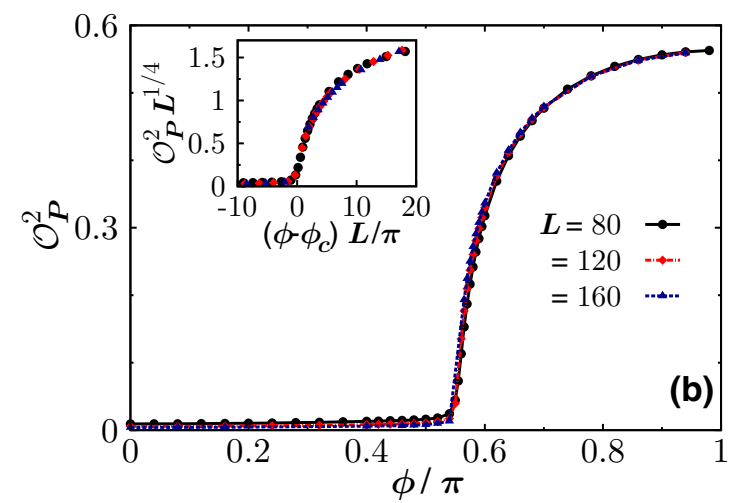

FIG. 12. Analysis of the transition from FM to SBF phase as a function of $\phi$ for $M=2, \Omega / U=0.8$, and $n=0.1$. (a) Behavior of the fidelity susceptibility $\chi_{F} / L^{2}$ as a function of $\phi$ is shown here. The inset shows the linear scaling of the peak height $\left(\chi_{F}^{\max } / L^{2}\right)$ with the system size. (b) Averaged parity order $\mathcal{O}_{P}^{2}$ as a function of $\phi$. The collapse of the data points is shown in the inset $\left(\phi_{c} \approx 0.55 \pi\right)$. 

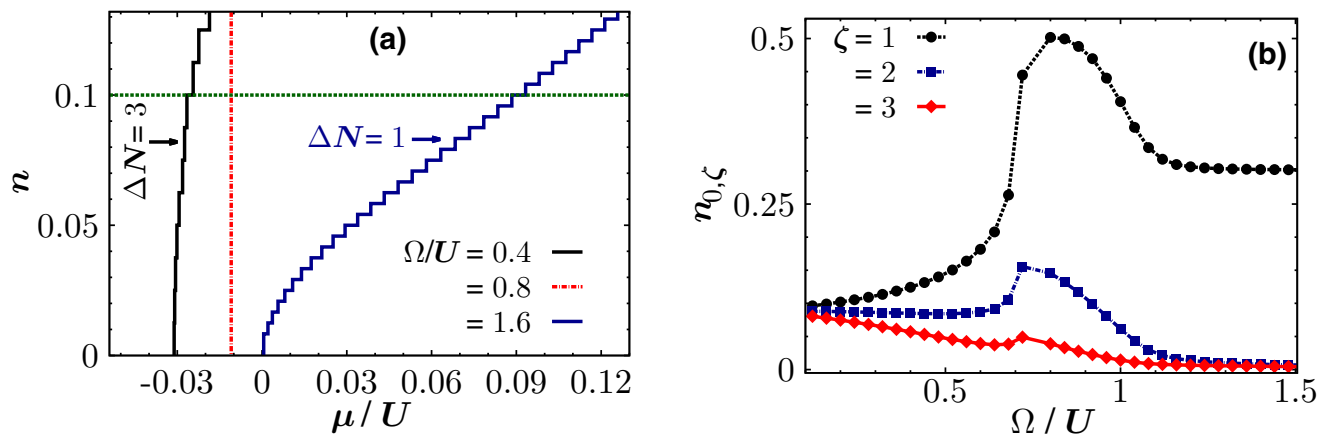

FIG. 13. Phase separation $(M=3)$ : Considering a particular flux $\phi / \pi=0.6$, we show phase separation in the $M=3$ SD system for $t / U=0.5, n=0.1$, and $L=80$. (a) Behavior of the equation of state of the system, i.e., the density $(n)$ as a function of the chemical potential $(\mu)$, for different values of $\Omega$. The chemical potential $\mu$ has been shifted for clarity. The green line shows the density $n=0.1$ under consideration and the $\Delta N$ 's show the steps in the number of particle $N$. (b) Variation of the average occupation at the central site $n_{0, \zeta}$ as a function of $\Omega$.

system as obtained from DMRG calculations by minimizing the functional $E_{0}(N, L)-\mu N$ [75]. While the 3-BF and FM phases are characterized by a series of steps in the number of particles $N, \Delta N=3$, and $\Delta N=1$, respectively [74], we note that there exists a regime where $\mu=$ const, i.e., a small change in the chemical potential produces a large change in the density. Hence, according to the definition of the compressibility $\kappa^{-1}=n^{2}\left(\frac{\partial \mu}{\partial n}\right)$, the system is infinitely compressible in this regime. As a result, as discussed in the text, particles tend to cluster near the central site at $L / 2$ and there is phase separation in the system. We further note from the behavior of the average occupation at the central site $n_{0, \zeta} \equiv\left\langle n_{i_{0}, \zeta}\right\rangle$ shown in Fig. 13(b) that with the increase in $\Omega$ from zero there is a dramatic increase in $n_{0, \zeta}$ for $\Omega$ values in the phase separation regime.

The phenomenon of phase separation can also be understood by looking at the noninteracting single-particle band structure of the system. It is noted that in the phase separation region the energy bands of the system become extremely flat (for a discussion of interesting phenomena occurring in systems with flat bands see also Refs. [76,77]). As a result, the kinetic energy of the system is frustrated and the system can only gain energy from the nonlocal induced interaction energy by clustering the particles. The system thus phase separates with high density of particles near the central site. We expect phase separation to occur for $M>3$ SD systems as well.

\section{APPENDIX C: CHIRAL CURRENTS}

Recent experimental works on ladder systems [78] and SD systems [16,17] focused on the study of chiral (also called boundary or edge) currents. Also, theoretically the chiral currents have been suggested to be very interesting observables to characterize various quantum phases [79-83]. From this viewpoint, we now discuss the behaviors of the chiral currents for the SD system with $\mathrm{SU}(M)$ symmetric attractive interactions.

From the continuity equation, we can define a current $J(\mathbf{r} \rightarrow \mathbf{s})$ from a site $\mathbf{r}$ to a neighboring site $\mathbf{s}$ for a system with the Hamiltonian $\mathcal{H}$ as $\left\langle\frac{d n_{\mathbf{r}}}{d t}\right\rangle=\mathrm{i}\left\langle\left[\mathcal{H}, n_{\mathbf{r}}\right]\right\rangle=-\sum_{\langle\mathbf{s}\rangle} J(\mathbf{r} \rightarrow \mathbf{s})$, where $n_{\mathbf{r}}$ is the density at site $\mathbf{r}$. In particular, in the original basis [see Eq. (1)] we define current for the SD system as

$$
J[(i, \gamma) \rightarrow(i+1, \gamma)]=\mathrm{i} t\left(C_{i+1, \gamma}^{\dagger} C_{i, \gamma}-C_{i, \gamma}^{\dagger} C_{i+1, \gamma}\right)
$$

The configuration of the local currents can give important insights into the properties of a quantum phase, but the average current that circulates through the boundary of a system, called chiral current, can give more interesting information. From the spin-resolved momentum distribution, this chiral current can be measured experimentally in typical setups $[16,17]$ and is defined as

$$
\begin{aligned}
J_{c}= & \frac{1}{L} \sum_{i}[\langle J[(i, 1) \rightarrow(i+1,1)]\rangle \\
& -\langle J[(i, M) \rightarrow(i+1, M)]\rangle] .
\end{aligned}
$$

Using the Hellman-Feynman theorem, we can compute $J_{c}$ from a derivative of the ground-state energy [79,80] $E_{0}$ and for the SD system

$$
J_{c}^{M=2}=\partial_{\phi} E_{0} / L \quad \text { and } \quad J_{c}^{M=3}=2 \partial_{\phi} E_{0} / L .
$$

In Figs. 14(a) and 14(b), we present the chiral currents data for $M=2$ and 3, respectively, corresponding to the phase diagrams in Figs. 6 and 7. For both the cases, $J_{c}$ is strongly suppressed in the bound-state phases. In the FM region, it increases approximately linearly with the flux and stays roughly constant as a function of $\Omega / U$. This feature allows us to interpret the FM phase to be a Meissner-like phase [56]. We can also identify some of the phase transitions by the kinks in the chiral current. In particular, during the FM to SBF transition, $J_{c}$ exhibits a strong drop and becomes slightly negative in the the SBF phase.

Figure 14(c) illustrates in detail the structures of some of the local current configurations for $M=2$. In the FM phase, as well as in the $2-\mathrm{BF}$ phase, the currents circulate through the boundary of the system, currents along the synthetic direction being strongly suppressed. Interestingly, the FFLO phase (where $c=2$ ) looks like a vortex phase with several vortices. 

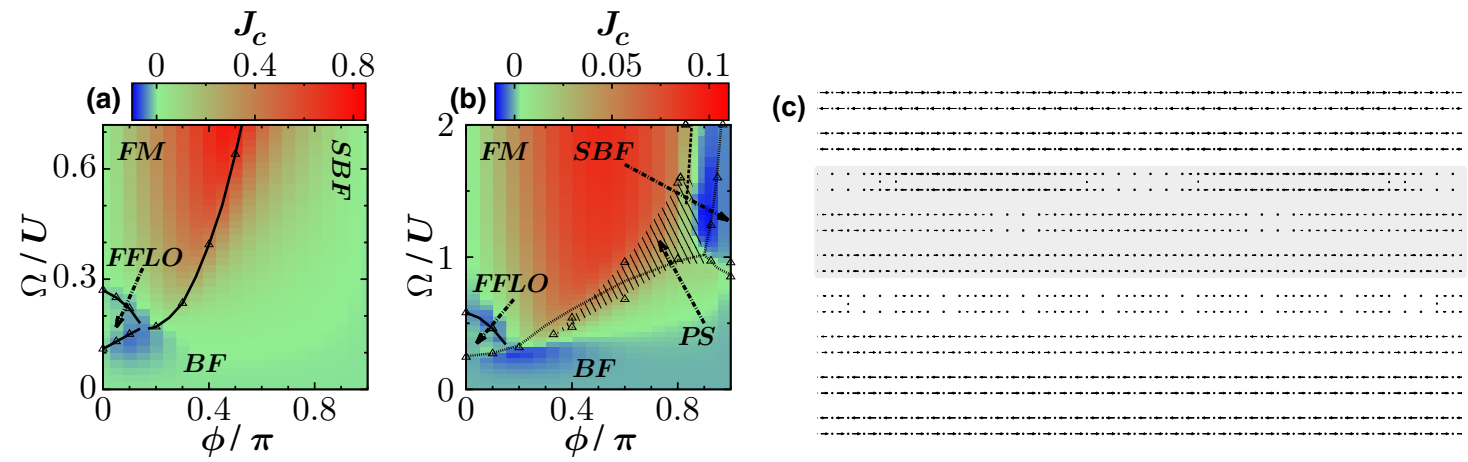

FIG. 14. Chiral current $J_{c}$ with $n=0.1$ and $t / U=0.5$ for (a) $M=2$ ( $L=80$ rungs) and (b) $M=3$ ( $L=40$ rungs). Solid lines are guides to the eye, dotted lines are the numerical estimates of bound-state transitions in the dilute limit, and the dashed lines correspond to the Lifshitz transition. (c) Examples of the local current configurations in the original basis [Eq. (1)] of $M=2 \mathrm{with} \phi / \pi=0.05$ and (top to bottom) $\Omega / U=0.08,0.12,0.16,0.2,0.24,0.28,0.36,0.44$, and 0.5 . The shading marks the current configurations corresponding to the $c=2$ phase.

[1] V. Mourik, K. Zuo, S. M. Frolov, S. R. Plissard, E. P. A. M. Bakkers, and L. P. Kouwenhoven, Science 336, 1003 (2012).

[2] T. D. Stanescu and S. Tewari, J. Phys.: Condens. Matter 25, 233201 (2013)

[3] M. I. Katsnelson and G. E. Volovik, J. Low Temp. Phys. 175, 655 (2014)

[4] J. Dalibard, F. Gerbier, G. Juzeliūnas, and P. Öhberg, Rev. Mod. Phys. 83, 1523 (2011).

[5] Y.-J. Lin, R. L. Compton, K. Jimenez-Garcia, J. V. Porto, and I. B. Spielman, Nature (London) 462, 628 (2009).

[6] L. W. Cheuk, A. T. Sommer, Z. Hadzibabic, T. Yefsah, W. S. Bakr, and M. W. Zwierlein, Phys. Rev. Lett. 109, 095302 (2012).

[7] J. Struck, C. Ölschläger, M. Weinberg, P. Hauke, J. Simonet, A. Eckardt, M. Lewenstein, K. Sengstock, and P. Windpassinger, Phys. Rev. Lett. 108, 225304 (2012).

[8] M. Aidelsburger, M. Atala, M. Lohse, J. T. Barreiro, B. Paredes, and I. Bloch, Phys. Rev. Lett. 111, 185301 (2013).

[9] H. Miyake, G. A. Siviloglou, C. J. Kennedy, W. C. Burton, and W. Ketterle, Phys. Rev. Lett. 111, 185302 (2013).

[10] L. Duca, T. Li, M. Reitter, I. Bloch, M. Schleier-Smith, and U. Schneider, Science 347, 288 (2015).

[11] N. Goldman, G. Juzeliunas, P. Ohberg, and I. B. Spielman, Rep. Prog. Phys. 77, 126401 (2014).

[12] W. S. Bakr, J. I. Gillen, A. Peng, S. Folling, and M. Greiner, Nature (London) 462, 74 (2009).

[13] M. Endres, M. Cheneau, T. Fukuhara, C. Weitenberg, P. Schauß, C. Gross, L. Mazza, M. C. Bañuls, L. Pollet, I. Bloch, and S. Kuhr, Appl. Phys. B 113, 27 (2013).

[14] A. Celi, P. Massignan, J. Ruseckas, N. Goldman, I. B. Spielman, G. Juzeliūnas, and M. Lewenstein, Phys. Rev. Lett. 112, 043001 (2014).

[15] J. K. Jain, Composite Fermions (Cambridge University Press, Cambridge, England, 2007).

[16] B. K. Stuhl, H.-I. Lu, L. M. Aycock, D. Genkina, and I. B. Spielman, Science 349, 1514 (2015).

[17] M. Mancini, G. Pagano, G. Cappellini, L. Livi, M. Rider, J. Catani, C. Sias, P. Zoller, M. Inguscio, M. Dalmonte, and L. Fallani, Science 349, 1510 (2015)
[18] L. F. Livi, G. Cappellini, M. Diem, L. Franchi, C. Clivati, M. Frittelli, F. Levi, D. Calonico, J. Catani, M. Inguscio, and L. Fallani, Phys. Rev. Lett. 117, 220401 (2016).

[19] S. Taie, R. Yamazaki, S. Sugawa, and Y. Takahashi, Nat. Phys. 8, 825 (2012).

[20] A. V. Gorshkov, M. Hermele, V. Gurarie, C. Xu, P. S. Julienne, J. Ye, P. Zoller, E. Demler, M. D. Lukin, and A. M. Rey, Nat. Phys. 6, 289 (2010).

[21] G. Pagano, M. Mancini, G. Cappellini, P. Lombardi, S. Schäfer, H. Hu, X.-J. Liu, J. Catani, C. Sias, M. Inguscio, and L. Fallani, Nat. Phys. 10, 198 (2014).

[22] T. Fukuhara, Y. Takasu, M. Kumakura, and Y. Takahashi, Phys. Rev. Lett. 98, 030401 (2007).

[23] B. J. DeSalvo, M. Yan, P. G. Mickelson, Y. N. Martinez de Escobar, and T. C. Killian, Phys. Rev. Lett. 105, 030402 (2010).

[24] M. Cazalilla and A. Rey, Rep. Prog. Phys. 77, 124401 (2014).

[25] R. Zhang, Y. Cheng, H. Zhai, and P. Zhang, Phys. Rev. Lett. 115, 135301 (2015).

[26] M. Höfer, L. Riegger, F. Scazza, C. Hofrichter, D. R. Fernandes, M. M. Parish, J. Levinsen, I. Bloch, and S. Fölling, Phys. Rev. Lett. 115, 265302 (2015).

[27] G. Pagano, M. Mancini, G. Cappellini, L. Livi, C. Sias, J. Catani, M. Inguscio, and L. Fallani, Phys. Rev. Lett. 115, 265301 (2015).

[28] T.-S. Zeng, C. Wang, and H. Zhai, Phys. Rev. Lett. 115, 095302 (2015).

[29] S. Barbarino, L. Taddia, D. Rossini, L. Mazza, and R. Fazio, Nat. Commun. 6, 8134 (2015).

[30] T. Bilitewski and N. R. Cooper, Phys. Rev. A 94, 023630 (2016).

[31] S. Greschner, M. Piraud, F. Heidrich-Meisner, I. P. McCulloch, U. Schollwöck, and T. Vekua, Phys. Rev. A 94, 063628 (2016).

[32] Z. Yan, S. Wan, and Z. Wang, Sci. Rep. 5, 15927 (2015).

[33] S. K. Ghosh, U. K. Yadav, and V. B. Shenoy, Phys. Rev. A 92, 051602(R) (2015).

[34] S. K. Ghosh and U. K. Yadav, Phys. Rev. A 94, 043634 (2016).

[35] G. Klingschat and C. Honerkamp, Phys. Rev. B 82, 094521 (2010).

[36] S. Capponi, G. Roux, P. Lecheminant, P. Azaria, E. Boulat, and S. R. White, Phys. Rev. A 77, 013624 (2008). 
[37] A. Rapp, G. Zaránd, C. Honerkamp, and W. Hofstetter, Phys. Rev. Lett. 98, 160405 (2007).

[38] J. Pohlmann, A. Privitera, I. Titvinidze, and W. Hofstetter, Phys. Rev. A 87, 023617 (2013).

[39] X.-W. Guan, M. T. Batchelor, and C. Lee, Rev. Mod. Phys. 85, 1633 (2013).

[40] M. D. Hoffman, A. C. Loheac, W. J. Porter, and J. E. Drut, arXiv:1609.01702 (2016).

[41] V. B. Shenoy, D. D. Sarma, and C. N. R. Rao, Chem. Phys. Chem. 7, 2053 (2006).

[42] T. Graß, R. W. Chhajlany, C. A. Muschik, and M. Lewenstein, Phys. Rev. B 90, 195127 (2014).

[43] A. O. Gogolin, A. A. Nersesyan, and A. M. Tsvelik, Bosonization and Strongly Correlated Systems (Cambridge University Press, Cambridge, England, 1999).

[44] T. Giamarchi, Quantum Physics in One Dimension (Clarendon, Oxford, 2004).

[45] E. Miranda, Braz. J. Phys. 33, 3 (2003).

[46] S. R. White, Phys. Rev. Lett. 69, 2863 (1992).

[47] S. R. White, Phys. Rev. B 48, 10345 (1993).

[48] U. Schollwöck, Rev. Mod. Phys. 77, 259 (2005).

[49] A. Luther and V. J. Emery, Phys. Rev. Lett. 33, 589 (1974).

[50] M. Di Liberto, C. E. Creffield, G. I. Japaridze, and C. Morais Smith, Phys. Rev. A 89, 013624 (2014).

[51] L. Cardarelli, S. Greschner, and L. Santos, Phys. Rev. A 94, 023615 (2016).

[52] G. Vidal, J. I. Latorre, E. Rico, and A. Kitaev, Phys. Rev. Lett. 90, 227902 (2003).

[53] P. Calabrese and J. Cardy, J. Stat. Mech.: Theory Exp. (2004) P06002.

[54] P. Calabrese and J. Cardy, J. Phys. A 42, 504005 (2009).

[55] S. T. Carr, B. N. Narozhny, and A. A. Nersesyan, Phys. Rev. B 73, 195114 (2006).

[56] E. Orignac and T. Giamarchi, Phys. Rev. B 64, 144515 (2001).

[57] A. Petrescu and K. Le Hur, Phys. Rev. Lett. 111, 150601 (2013).

[58] A. Petrescu and K. Le Hur, Phys. Rev. B 91, 054520 (2015).

[59] A. E. Feiguin, F. Heidrich-Meisner, G. Orso, and W. Zwerger, J. Lect. Notes Phys. 836, 503 (2011).

[60] I. M. Lifshitz, Sov. Phys. JETP 11, 1130 (1930).

[61] Y. Blanter, M. Kaganov, A. Pantsulaya, and A. Varlamov, Phys. Rep. 245, 159 (1994).

[62] M. Bartenstein, A. Altmeyer, S. Riedl, R. Geursen, S. Jochim, C. Chin, J. H. Denschlag, R. Grimm, A. Simoni, E. Tiesinga,
C. J. Williams, and P. S. Julienne, Phys. Rev. Lett. 94, 103201 (2005).

[63] M. Ben Dahan, E. Peik, J. Reichel, Y. Castin, and C. Salomon, Phys. Rev. Lett. 76, 4508 (1996).

[64] M. Köhl, H. Moritz, T. Stöferle, K. Günter, and T. Esslinger, Phys. Rev. Lett. 94, 080403 (2005).

[65] I. Bloch, J. Dalibard, and W. Zwerger, Rev. Mod. Phys. 80, 885 (2008).

[66] L. Tarruell, D. Greif, T. Uehlinger, G. Jotzu, and T. Esslinger, Nature (London) 483, 302 (2012).

[67] T. Kinoshita, T. Wenger, and D. S. Weiss, Phys. Rev. Lett. 95, 190406 (2005).

[68] R. Casalbuoni and G. Nardulli, Rev. Mod. Phys. 76, 263 (2004).

[69] W. Chen, K. Hida, and B. C. Sanctuary, Phys. Rev. B 67, 104401 (2003).

[70] A. J. Daley, J. M. Taylor, S. Diehl, M. Baranov, and P. Zoller, Phys. Rev. Lett. 102, 040402 (2009).

[71] S. Greschner, L. Santos, and T. Vekua, Phys. Rev. A 87, 033609 (2013).

[72] S.-J. GU, Int. J. Mod. Phys. B 24, 4371 (2010).

[73] S. Greschner, A. K. Kolezhuk, and T. Vekua, Phys. Rev. B 88, 195101 (2013).

[74] M. Azimi, L. Chotorlishvili, S. K. Mishra, S. Greschner, T. Vekua, and J. Berakdar, Phys. Rev. B 89, 024424 (2014).

[75] T. Mishra, S. Greschner, and L. Santos, Phys. Rev. A 91, 043614 (2015).

[76] S. D. Huber and E. Altman, Phys. Rev. B 82, 184502 (2010).

[77] M. Tovmasyan, S. Peotta, P. Törmä, and S. D. Huber, Phys. Rev. B 94, 245149 (2016).

[78] M. Atala, M. Aidelsburger, M. Lohse, J. T. Barreiro, B. Paredes, and I. Bloch, Nat. Phys. 10, 588 (2014).

[79] M. Piraud, F. Heidrich-Meisner, I. P. McCulloch, S. Greschner, T. Vekua, and U. Schollwöck, Phys. Rev. B 91, 140406 (2015).

[80] S. Greschner, M. Piraud, F. Heidrich-Meisner, I. P. McCulloch, U. Schollwöck, and T. Vekua, Phys. Rev. Lett. 115, 190402 (2015)

[81] F. Kolley, M. Piraud, I. P. McCulloch, U. Schollwöck, and F. Heidrich-Meisner, New J. Phys. 17, 092001 (2015).

[82] S. Barbarino, L. Taddia, D. Rossini, L. Mazza, and R. Fazio, New J. Phys. 18, 035010 (2016).

[83] E. Cornfeld and E. Sela, Phys. Rev. B 92, 115446 (2015). 